\title{
Concealed Infective Endocarditis Associated with Subaortic Left Ventricular Aneurysm
}

\author{
Akihiko Ikeda ${ }^{1}$ Shonosuke Matsushita ${ }^{1}$ Yuzuru Sakakibara ${ }^{1}$ \\ ${ }^{1}$ Department of Cardiovascular Surgery, Graduate School of \\ Comprehensive Human Sciences, University of Tsukuba, Tsukuba, \\ Japan \\ Thorac Cardiovasc Surg 2012;60(S2):e9-e12. \\ Address for correspondence Dr. Yuzuru Sakakibara, M.D., \\ Department of Cardiovascular Surgery, Graduate School of \\ Comprehensive Human Sciences, University of Tsukuba, \\ 1-1-1 Tennodai, Tsukuba, Ibaraki 305-8575, Japan \\ (e-mail: kensan@md.tsukuba.ac.jp).
}

\author{
Abstract \\ Keywords \\ - endocarditis \\ - subvalvular aneurysm \\ - aortic valve \\ replacement
}

We describe a case of subaortic left ventricular aneurysm with concealed infective endocarditis. The patient, who was diagnosed with aortic regurgitation and a subaortic left ventricular aneurysm, did not exhibit any evidence of infective endocarditis preoperatively. However, histopathological examination after an aortic valve replacement revealed neutrophil infiltration in the resected aneurysm. One year postoperatively, the infection recurred and an aortic root replacement was performed.
Subvalvular left ventricular aneurysm is a rare entity. Infective endocarditis, which is one of the causes of subvalvular left ventricular aneurysm, usually displays typical findings, such as fever, leucocytosis, and positive blood culture, and is diagnosed before surgery. However, because of the absence of such findings, latent infective endocarditis is only first diagnosed after surgery, upon histopathological examination. This is a case report describing a subaortic left ventricular aneurysm associated with latent infective endocarditis.

\section{Case Report}

A 47-year-old woman was referred to our hospital for treatment of aortic regurgitation (AR) and an abnormal structure of the left ventricular outflow tract (LVOT). The patient did not exhibit fever. Neither was there evidence of leucocytosis nor elevation of C-reactive protein (CRP), even at the previous hospital. Two-dimensional echocardiography showed that an aneurysm, which had a few saccular structures inside, extended from the right coronary cusp (RCC) to the LVOT. This aneurysm arose from the thinned basal wall of the interventricular septum and the RCC had a fistula which communicated with this aneurysm (-Fig. 1). Upon Doppler echocardiography, subaortic stenosis (a peak pressure gradient of $88 \mathrm{~mm} \mathrm{Hg}$ across the LVOT obstruction) and severe AR were detected. One AR flow passed through the aneurysm and reached the left ventricular cavity. In addition, another AR flow, caused by a prolapsed RCC, was detected at the

received

July 2, 2011

accepted after revision

August 2, 2011

published online

January 3, 2012

central commissure of the aortic valve. On the basis of the echocardiographic findings, infective endocarditis was suspected as a differential diagnosis. However, one set of blood cultures was negative.

A diagnosis of subaortic stenosis and severe AR caused by a subaortic left ventricular aneurysm was made, and an aortic valve replacement (AVR) was performed. At surgery, three cusps of the aortic valve were even and slightly thickened. The RCC had a fistula with a diameter of $8 \mathrm{~mm}$ at the aortic annulus and this fistula communicated with a subaortic left ventricular aneurysm. This aneurysm was placed at the interventricular septum and perforated into the left ventricular cavity. However, there were no signs of vegetation. After resection of the left and noncoronary cusps, the RCC and aneurysm were resected en bloc (-Fig. 2). Finally, a 21-mm mechanical prosthesis (On-X Life Technologies, Inc., Austin, Texas) was attached to the aortic annulus.

Routine antibiotic treatment (cefazorin: $1 \mathrm{~g} / 8$ hours), initiated 30 minutes before surgery, was discontinued 48 hours after surgery. Definitive histopathological findings revealed the infiltration of neutrophils in the wall of the resected aneurysm (-Fig. 3). The patient underwent a 4-week course of antibiotic therapy for infective endocarditis (Penicillin G: 180 million units/day), although the staining and cultures of the resected tissues did not reveal any microorganisms. Eight weeks postoperatively, the patient was discharged with a prescription for orally administered cefdinir (300 mg/day). At that time, her leukocyte counts and CRP

(c) 2012 Georg Thieme Verlag KG Stuttgart · New York
DOI http://dx.doi.org/ 10.1055/s-0031-1295583. ISSN 2194-7635. 


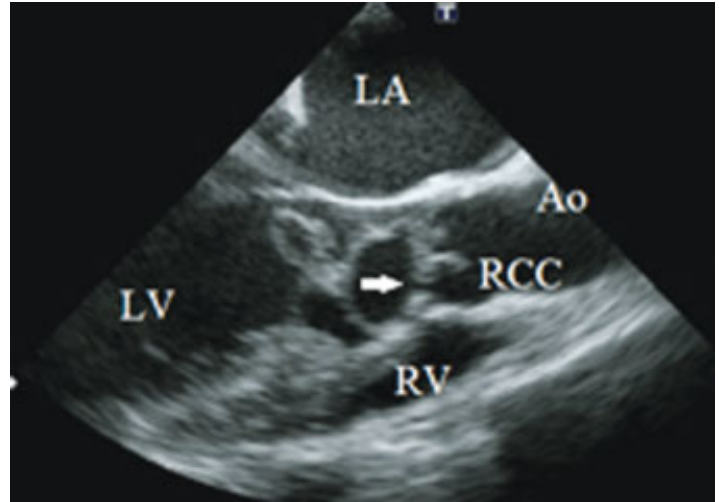

Figure 1 Transesophageal echocardiography shows a subaortic aneurysm consisting of a few cystic structures, protruding into the left ventricular outflow tract. The RCC has a fistula which communicates with this subaortic aneurysm (white arrow). (LA, left atrium; Ao, aorta; $\mathrm{RV}$, right ventricle; LV, left ventricle.)

were not elevated. In addition, no paravalvular leakage was detected on Doppler echocardiography.

However, 1 year postoperatively, this patient was admitted to our hospital again due to severe heart failure with massive paravalvular leakage. A diagnosis of prosthetic valvular endocarditis was made and urgent surgery was performed. Because the aortic annulus and its adjacent tissue were destroyed by the infection, debridement over a wide area was necessary. After debridement, an aortic root replacement was performed by using a composite graft which was made of a 21-mm mechanical prosthesis (ATS Medical, Inc., Minneapolis, Minnesota) and a 24-mm woven Dacron graft (HEMASHIELD GOLD, MAQUET, Inc., Wayne, New Jersey). The staining and cultures of the resected tissues did not reveal any microorganisms. In addition, preoperative blood cultures were also negative.

After surgery, she was treated with vancomycin, gentamycin, and rifampicin. However, her medication was changed to linezolid because of drug-induced erythema. Finally, she was discharged and prescribed linezolid to be taken orally. Although the treatment of linezolid had to be stopped after 7 months because of profound anemia, infection and congestive heart failure have not recurred.

\section{Discussion}

Subvalvular left ventricular aneurysm is a rare entity which was first reported in $1962 .{ }^{1}$ This type of aneurysm usually arises from the annular subaortic or submitral region of the left ventricle, presumably due to weakness of the fibromuscular annuli, and should be differentiated from other aneurysms, such as valve aneurysm, sinus of Valsalva aneurysm, and left ventricular pseudoaneurysm. ${ }^{2}$ Although the etiology of subvalvular left ventricular aneurysm is uncertain, several infectious diseases, such as infective endocarditis, tuberculosis, and rheumatic fever, have been reported as possible causes of subvalvular left ventricular aneurysm. ${ }^{3-5}$

In this case, it was considered that the subaortic aneurysm was caused by infective endocarditis because histopathological examination showed neutrophil infiltration in the wall of the subaortic aneurysm after the first operation. Neutrophil infiltration, which usually suggests the presence of acute inflammation, is one of the histopathological findings of infective endocarditis. ${ }^{6}$

However, the typical findings of endocarditis, such as fever, leucocytosis, elevation of CRP, and positive blood

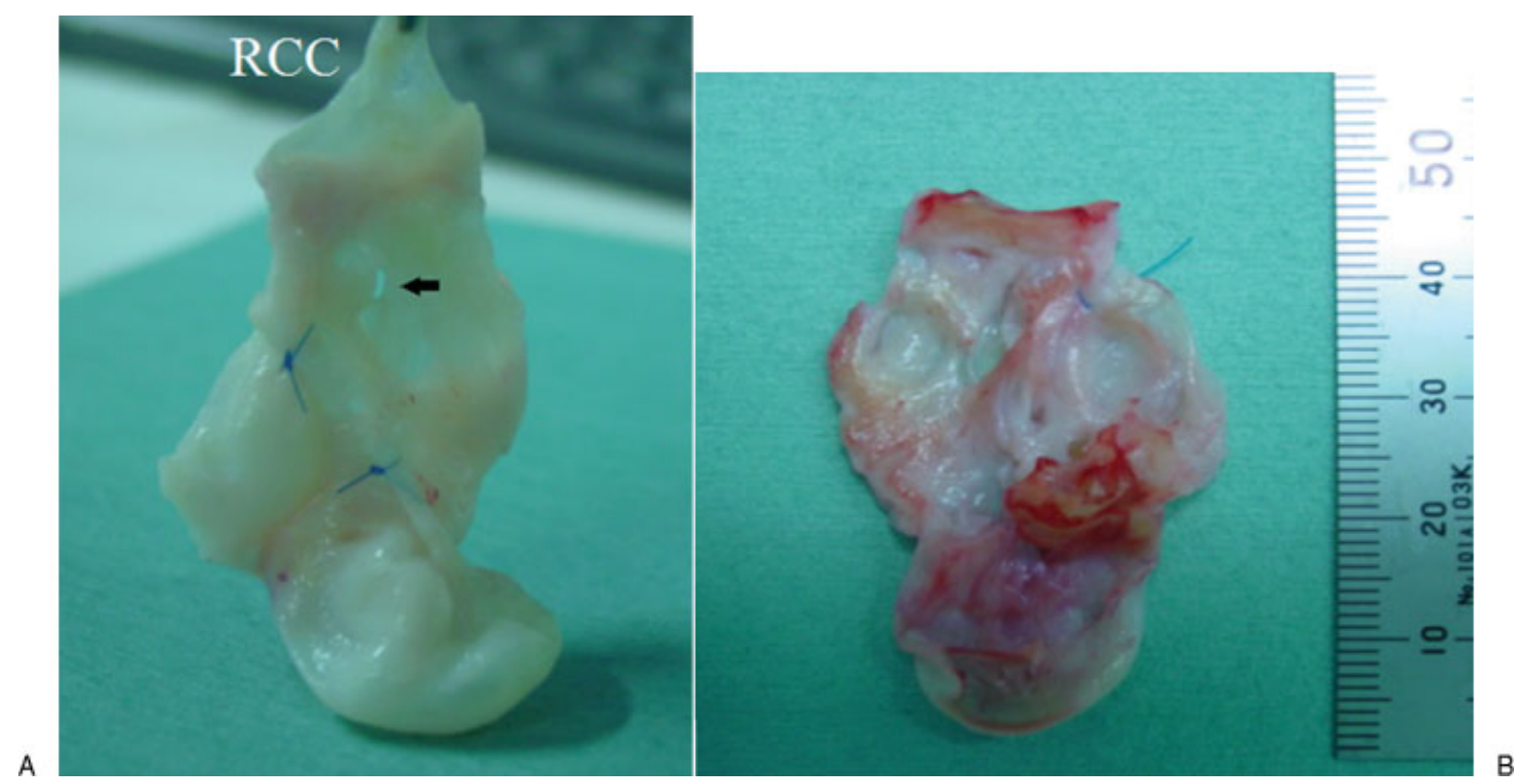

Figure 2 The RCC and a subaortic aneurysm resected at surgery. (A) Observed from the left ventricular cavity. The RCC adjoins a shrunken aneurysm. The black arrow indicates a perforation. (B) Observed from the reverse side. The inner surface of the aneurysm is rough and looks like a group of cystic lesions. 


\section{A}
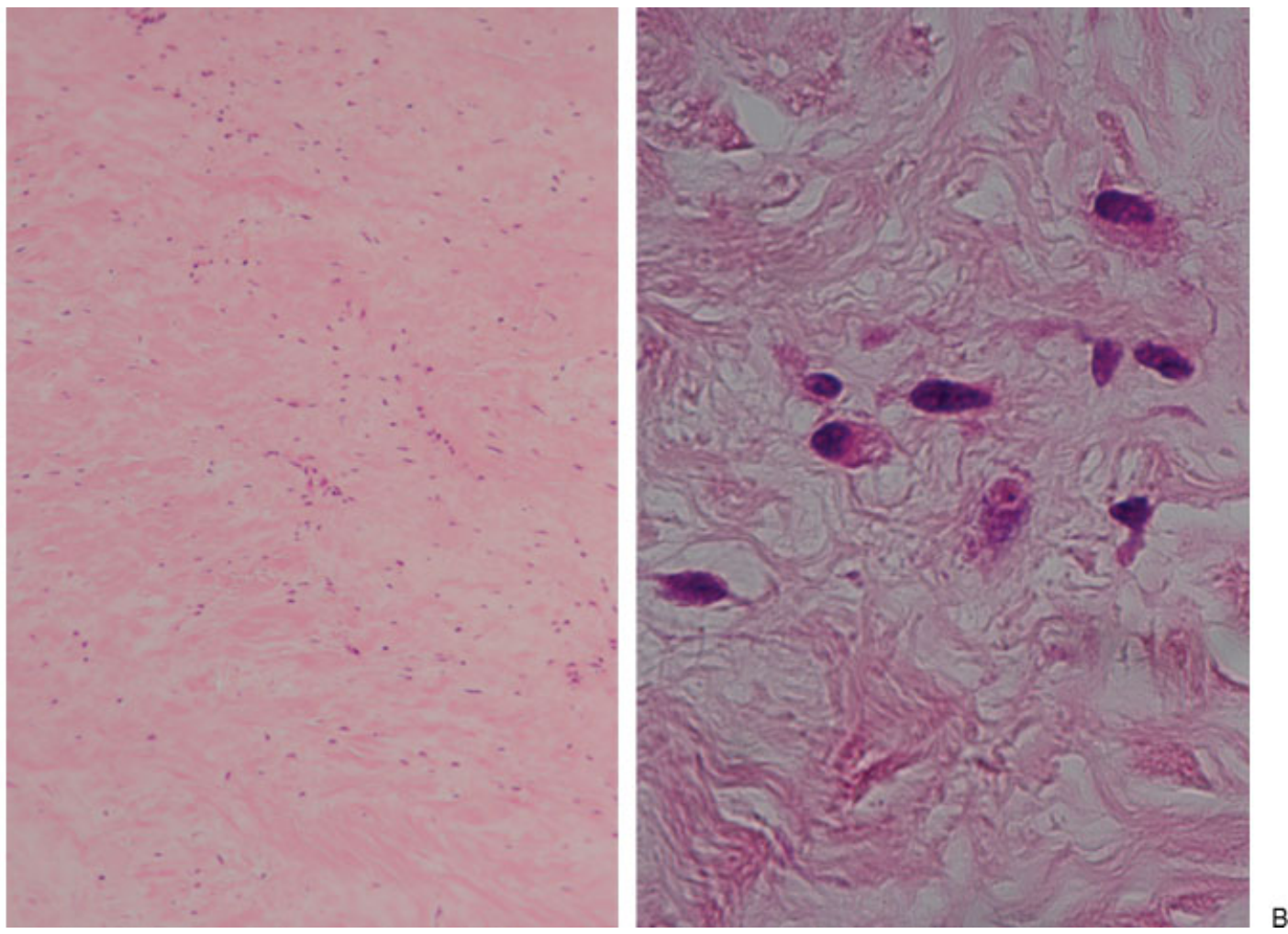

Figure 3 Histology of the resected aneurysm (Hematoxylin-Eosin stain, A: $\times 100, B: \times 1000)$. (A) Inflammatory cell infiltration is observed in the wall of the resected aneurysm. (B) A magnified photography shows the presence of neutrophils among the inflammatory cells.

cultures were not observed before AVR in this case. This condition has been previously reported as latent infective endocarditis, which has a high incidence of recurrent infection involving newly placed prosthetic valves $(25 \%){ }^{7}$ One of the reasons for this is the delay in the onset of antibiotic therapy for infective endocarditis because it takes a certain period of time to make a histopathological diagnosis. As in our case, there was a blank period during which the patient was not administered any antibiotics and, consequently, antibiotic therapy after histopathological diagnosis could not prevent prosthetic valvular endocarditis. In most cases, treatment for subvalvular left ventricular aneurysm requires prosthetic valve replacement due to complicated valve insufficiency. Thus, it is important to suspect the presence of latent infective endocarditis when patients with subvalvular left ventricular aneurysm are treated. If a prosthetic valve replacement is needed, sufficient preemptive antibiotic therapy, which is continued from a preoperative period until a definitive histopathological diagnosis is made, is essential to prevent infection of newly implanted prosthetic valves.

\section{Conclusion}

We performed an AVR for a patient with a subaortic left ventricular aneurysm associated with latent infective endocarditis. Infective endocarditis is one of the causes of sub- valvular left ventricular aneurysm and is rarely latent, as our case was. We suggest that prophylactic preemptive antibiotic therapy, continued from a preoperative period until definitive histopathological diagnosis is made, is essential to prevent recurrent infection when patients with subvalvular left ventricular aneurysm need a prosthetic valve replacement, even if typical findings of infective endocarditis cannot be observed preoperatively.

\section{Acknowledgments}

The authors are grateful to Mr. Avi Landau for his language consultation.

\section{References}

1 Abrahams DG, Barton CJ, Cockshott WP, Edington GM, Weaver EJ. Annular subvalvular left ventricular aneurysms. Q J Med 1962; 31;345-360

2 Chesler E, Joffe N, Schamroth L, Meyers A. Annular subvalvular left ventricular aneurysms in the South African Bantu. Circulation 1965;32;43-51

3 Deshpande J, Vaideeswar P, Sivaraman A. Subvalvular left ventricular aneurysms. Cardiovasc Pathol 2000;9(5):267-271

4 Sogabe O, Ohya T. A case of tuberculous endocarditis with acute aortic valve insufficiency and annular subvalvular left ventricular aneurysm. Gen Thorac Cardiovasc Surg 2007;55 (2):61-64 
5 Müller H, Cikirikcioglu M, Lerch R. Subaortic aneurysm caused by Paecilomyces lilacinus endocarditis. Arch Cardiovasc Dis 2008;101 (11-12):803-804

6 McGiffin DC, Galbraith AJ, McLachlan GJ, et al. Aortic valve infection. Risk factors for death and recurrent endocarditis after aortic valve replacement. J Thorac Cardiovasc Surg 1992;104 (2):511-520
7 Shapira N, Merin O, Rosenmann E, et al. Latent infective endocarditis: epidemiology and clinical characteristics of patients with unsuspected endocarditis detected after elective valve replacement. Ann Thorac Surg 2004;78(5):1623-1629 